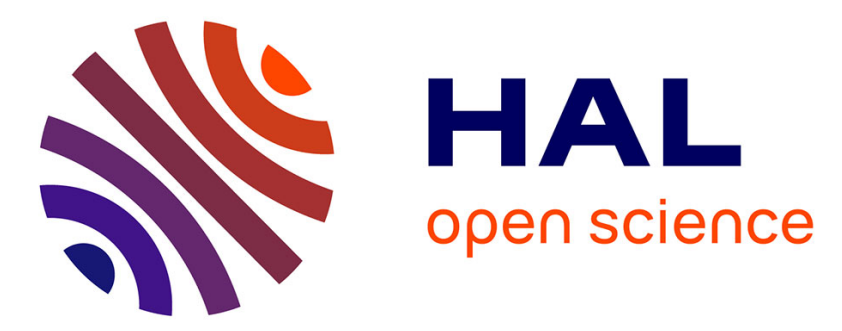

\title{
Focusing of light through a stratified medium: a practical approach for computing fluorescence microscope point spread functions. Part II: confocal and multiphoton microscopy
}

Olivier Haeberle

\section{To cite this version:}

Olivier Haeberle. Focusing of light through a stratified medium: a practical approach for computing fluorescence microscope point spread functions. Part II: confocal and multiphoton microscopy. Optics Communications, 2004, 235, pp.1-10. 10.1016/j.optcom.2004.02.068 . hal-00934825

\author{
HAL Id: hal-00934825 \\ https://hal.science/hal-00934825
}

Submitted on 24 Feb 2014

HAL is a multi-disciplinary open access archive for the deposit and dissemination of scientific research documents, whether they are published or not. The documents may come from teaching and research institutions in France or abroad, or from public or private research centers.
L'archive ouverte pluridisciplinaire HAL, est destinée au dépôt et à la diffusion de documents scientifiques de niveau recherche, publiés ou non, émanant des établissements d'enseignement et de recherche français ou étrangers, des laboratoires publics ou privés. 


\title{
Focusing of light through a stratified medium: a practical
} approach for computing microscope point spread

\section{functions. Part II: confocal and multiphoton microscopy}

\author{
Olivier Haeberlé ${ }^{1}$ \\ Groupe LabEl - Laboratoire MIPS, Université de Haute-Alsace IUT Mulhouse, \\ 61 rue A. Camus F-68093 Mulhouse Cedex France
}

\begin{abstract}
:
We propose a rigorous and easy to use model to compute point spread functions of confocal and multiphoton microscopes imaging through a stratified medium. Our model is based on vectorial theories for illumination and detection, combined with the simple raytracing method of Gibson and Lanni to calculate the aberration function. We show the validity of this approach, which is always very precise for biological applications, and exact in the case of a single interface. For mediums with very large index of refraction gaps, small discrepancies appear between rigorous vectorial theories and our simplified model. Our model may also be used to precisely compute point spread functions of 4-Pi and STED microscopes.
\end{abstract}

PACS numbers: $\quad$ 07.60.Pb, 42.25.Fx, 42.30.Va

Keywords: Optical microscopy, Focusing, Point Spread Function, Vectorial theory

${ }^{1}$ o.haeberle@uha.fr 


\section{Introduction}

The optical microscope has proven to be an invaluable tool in fields as diverse as biology, surface and solid-state physics, metallurgy, MEMs or Integrated Circuit inspection. A correct understanding of the image formation mechanism is mandatory to further improve the optics, master the data acquisition process, or correctly process the acquired images by software.

In order to take into account polarization effects, vectorial theories are needed to correctly describe the propagation of light towards the specimen (as for example to model the illumination in a confocal microscope), as well as to describe the collection by the objective of the light emitted by the specimen.

In biology, the specimen is often embedded between a glass and a cover glass. Vectorial models for focusing of light through a layered or stratified medium have been developed by several authors [1-6]. Recently, a vectorial theory for the detection of dipole emission through a stratified medium has been published $[7,8]$. Combining these approaches permits to describe the image formation process in a confocal microscope [9].

In this paper, we first briefly recall the vectorial theories for illumination and detection through a stratified medium. As in Ref. [10] for the illumination Point Spread Function (PSF), we show how to explicitly introduce experimental and design acquisition parameters, as recommended by the objective manufacturer, to compute the actual detection Point Spread Function. We then discuss the validity of the assumption used to combine the simple Gibson and Lanni method to calculate the aberration function [11] with vectorial models in the case of confocal and multiphoton microscopy, in view of biologic, crystallographic, and semiconductor applications.

Figure 1 sketches a confocal microscope imaging a specimen through an immersion medium and a cover glass. If the illumination of the specimen is considered as homogeneous, then Figure 1 depicts a wide field microscope. 


\section{Vectorial model for the illumination PSF}

We model now the illumination PSF in a confocal microscope. One considers a linearly polarized (along the x-axis) monochromatic wave focused through the immersion medium, the cover glass and the specimen (see Fig. 1). The origin of the (x,y,z) coordinate system is at the unaberrated Gaussian focus point. Following Török and Varga, the intensity illumination PSF at point $\mathrm{P}(\mathrm{x}, \mathrm{y}, \mathrm{z})$ can then be computed as $[6]$ :

$$
P S F_{i l l}(x, y, z)=|\mathbf{E}|^{2}=\left|\mathbf{E}_{3 x}+\mathbf{E}_{3 y}+\mathbf{E}_{3 z}\right|^{2}
$$

the components being given by (with $\phi$ in spherical polar coordinates):

$$
\begin{aligned}
E_{3 x}=-i\left(I_{0 i l l}\right. & \left.+I_{2 i l l} \cos 2 \phi\right), E_{3 y}=-i\left(I_{2 i l l} \sin 2 \phi\right), E_{3 z}=-2 I_{1 i l l} \cos \phi \\
I_{0 i l l} & =\int_{0}^{\alpha}\left(\cos \theta_{1}\right)^{1 / 2} \sin \theta_{1} J_{0}\left(k_{1}\left(x^{2}+y^{2}\right)^{1 / 2} \sin \theta_{1}\right) \\
& \times\left(T_{2 s}+T_{2 p} \cos \theta_{3}\right) \exp \left(i k_{0} \Psi_{i l l}\right) \exp \left(i k_{3} z \cos \theta_{3}\right) d \theta_{1} \\
I_{1 i l l}= & \int_{0}^{\alpha}\left(\cos \theta_{1}\right)^{1 / 2} \sin \theta_{1} J_{1}\left(k_{1}\left(x^{2}+y^{2}\right)^{1 / 2} \sin \theta_{1}\right) \\
& \times T_{2 p} \sin \theta_{3} \exp \left(i k_{0} \Psi_{i l l}\right) \exp \left(i k_{3} z \cos \theta_{3}\right) d \theta_{1} \\
I_{2 i l l}= & \int_{0}^{\alpha}\left(\cos \theta_{1}\right)^{1 / 2} \sin \theta_{1} J_{2}\left(k_{1}\left(x^{2}+y^{2}\right)^{1 / 2} \sin \theta_{1}\right) \\
& \times\left(T_{2 s}-T_{2 p} \cos \theta_{3}\right) \exp \left(i k_{0} \Psi_{i l l}\right) \exp \left(i k_{3} z \cos \theta_{3}\right) d \theta_{1}
\end{aligned}
$$

the so-called initial aberration function [6] being given by:

$$
\Psi_{i l l}=h_{2} n_{3} \cos \theta_{3}-h_{1} n_{1} \cos \theta_{1}
$$

The transmission coefficient for a three-layer medium is given by:

$$
T_{2 s, p}=\frac{t_{12 s, p} t_{23 s, p} \exp (i \beta)}{1+r_{12 s, p} r_{23 s, p} \exp (2 i \beta)}
$$

with $\beta=k_{2}\left|h_{2}-h_{1}\right| \cos \theta_{2}$ and the Fresnel coefficients for transmission and reflection for the illumination case are computed from medium $\mathrm{n}$ towards medium $\mathrm{n}+1$ : 


$$
\begin{aligned}
t_{n n+1, s} & =\frac{2 n_{n} \cos \theta_{n}}{n_{n} \cos \theta_{n}+n_{n+1} \cos \theta_{n+1}} \\
t_{n n+1, p} & =\frac{2 n_{n} \cos \theta_{n}}{n_{n+1} \cos \theta_{n}+n_{n} \cos \theta_{n+1}} \\
r_{n n+1, s} & =\frac{n_{n} \cos \theta_{n}-n_{n+1} \cos \theta_{n+1}}{n_{n} \cos \theta_{n}+n_{n+1} \cos \theta_{n+1}} \\
r_{n n+1, p} & =\frac{n_{n+1} \cos \theta_{n}-n_{n} \cos \theta_{n+1}}{n_{n+1} \cos \theta_{n}+n_{n} \cos \theta_{n+1}}
\end{aligned}
$$

\section{Vectorial model for the detection PSF}

We consider now a dipole, located near $\mathrm{O}$, which is excited by the illumination PSF. The moment is proportional to the excitation intensity $p_{e} \propto P S F_{i l l}$ (one-photon process), as given by Eqs. (1). We use a $\left(x^{\prime}, y^{\prime}, z^{\prime}\right)$ reference frame centered at the location of the dipole $\mathrm{O}^{\prime}$. The electric field emitted by the fluorescence traverses back the stratified medium. It is collimated by the lens and refocused onto the detector. The field on the detector is given by [8]:

$$
\begin{aligned}
& \mathrm{E}_{x^{\prime}}=p_{e x}^{*}\left(I_{0 \mathrm{det}}+I_{2 \mathrm{det}} \cos 2 \phi_{d}\right)+p_{e y}^{*}\left(I_{2 \mathrm{det}} \sin 2 \phi_{d}\right)-2 i I_{1 \mathrm{det}} p_{e z}^{*} \cos \phi_{d} \\
& \mathrm{E}_{y^{\prime}}=p_{e x}^{*} I_{2 \mathrm{det}} \sin 2 \phi_{d}+p_{e y}^{*}\left(I_{0 \mathrm{det}}-I_{2 \mathrm{det}} \cos 2 \phi_{d}\right)-2 i I_{1 \mathrm{det}} p_{e z}^{*} \sin \phi_{d}
\end{aligned}
$$

with the quantities $I_{0 \operatorname{det}}, I_{1 \text { det }}$ and $I_{2 \operatorname{det}}$ defined as:

$$
\begin{aligned}
I_{0 \mathrm{det}} & =\int_{0}^{\alpha_{d}}\left(\cos \theta_{1}^{\prime}\right)^{-1 / 2} \sin 2 \theta_{d} J_{0}\left(k_{d}\left(x^{\prime 2}+y^{\prime 2}\right)^{1 / 2} \sin \theta_{d}\right) \\
& \times\left(T_{s}^{\prime}+T_{p}^{\prime} \cos \theta_{3}^{\prime}\right) \exp \left(-i k_{0} \Psi_{\mathrm{det}}\right) \exp \left(-i k_{1} z^{\prime} \cos \theta_{1}^{\prime}\right) d \theta_{d} \\
I_{1 \mathrm{det}} & =\int_{0}^{\alpha_{d}}\left(\cos \theta_{1}^{\prime}\right)^{-1 / 2} \sin 2 \theta_{d} J_{1}\left(k_{d}\left(x^{\prime 2}+y^{\prime 2}\right)^{1 / 2} \sin \theta_{d}\right) \\
& \times T_{p}^{\prime} \sin \theta_{3}^{\prime} \exp \left(-i k_{0} \Psi_{\mathrm{det}}\right) \exp \left(-i k_{1} z^{\prime} \cos \theta_{1}^{\prime}\right) d \theta_{d} \\
I_{2 \mathrm{det}} & =\int_{0}^{\alpha_{d}}\left(\cos \theta_{1}^{\prime}\right)^{-1 / 2} \sin 2 \theta_{d} J_{2}\left(k_{d}\left(x^{\prime 2}+y^{\prime 2}\right)^{1 / 2} \sin \theta_{d}\right) \\
& \times\left(T_{s}^{\prime}-T_{p}^{\prime} \cos \theta_{3}^{\prime}\right) \exp \left(-i k_{0} \Psi_{\mathrm{det}}\right) \exp \left(-i k_{1} z^{\prime} \cos \theta_{1}^{\prime}\right) d \theta_{d}
\end{aligned}
$$


with $\alpha_{d}$ being the angular aperture of the detector lens, and the azimuthal angle $\theta_{d}$ being related to the azimuthal angle $\theta_{1}^{\prime}$ by the relationship:

$$
\frac{k_{1} \sin \alpha_{1}}{k_{d} \sin \alpha_{d}}=\frac{k_{1} \sin \theta_{1}^{\prime}}{k_{d} \sin \theta_{d}}=\beta
$$

where $\beta$ is the nominal magnification of the detector lens system, and $k_{\mathrm{d}}$ is the wave number in the image space. For a three-layered medium, the initial aberration function for detection is given by [8]:

$$
\Psi_{\text {det }}=n_{1} h_{1} \cos \theta_{1}^{\prime}-n_{3} h_{2} \cos \theta_{3}^{\prime}
$$

The detected intensity is then obtained as:

$$
P S F_{\text {det }}\left(x^{\prime}, y^{\prime}, z^{\prime}\right)=\left|\mathrm{E}_{x^{\prime}}\right|^{2}+\left|\mathrm{E}_{y^{\prime}}\right|^{2}
$$

For the detection, the transmission coefficient for a three-layer medium is given by:

$$
T_{2 s, p}^{\prime}=\frac{t_{32 s, p} t_{21 s, p} \exp (-i \beta)}{1+r_{32 s, p} r_{21 s, p} \exp (-2 i \beta)}
$$

with $\beta=k_{2}\left|h_{2}-h_{1}\right| \cos \theta_{2}^{\prime}$ and the Fresnel coefficients for transmission and reflection being now computed in the detection case from medium $n$ towards medium n-1 (i.e. in the opposite direction as for the illumination) as:

$$
\begin{aligned}
t_{n n-1, s} & =\frac{2 n_{n} \cos \theta_{n}^{\prime}}{n_{n} \cos \theta_{n}^{\prime}+n_{n-1} \cos \theta_{n-1}^{\prime}} \\
t_{n n-1, p} & =\frac{2 n_{n} \cos \theta_{n}^{\prime}}{n_{n-1} \cos \theta_{n}^{\prime}+n_{n} \cos \theta_{n-1}^{\prime}} \\
r_{n n-1, s} & =\frac{n_{n} \cos \theta_{n}^{\prime}-n_{n-1} \cos \theta_{n-1}^{\prime}}{n_{n} \cos \theta_{n}^{\prime}+n_{n-1} \cos \theta_{n-1}^{\prime}} \\
r_{n n-1, p} & =\frac{n_{n-1} \cos \theta_{n}^{\prime}-n_{n} \cos \theta_{n-1}^{\prime}}{n_{n-1} \cos \theta_{n}^{\prime}+n_{n} \cos \theta_{n-1}^{\prime}}
\end{aligned}
$$




\section{Approximation of the aberration function in biology}

In biological microscopy, one usually uses a dry objective, an oil immersion objective or a water immersion objective, the latter being the most interesting for 3-D investigations, the former being mostly used for inspection of large specimen. In these cases, it has been shown $[6,10]$ that the overall aberration function, consisting in the sum of the initial aberration function and the aberration due to the three-layer medium can be simplified as:

$$
\Psi_{i l l}=\sum_{j=1}^{3} h_{j}\left(n_{j+1} \cos \theta_{j+1}-n_{j} \cos \theta_{j}\right)
$$

The hypothesis leading to Eq. (14) is that for biological applications, the Fresnel reflection coefficients $r_{\mathrm{s}, \mathrm{p}}$ given by Eq. (13) are much smaller than unity so that the denominator of $T_{2 \mathrm{~s}, \mathrm{p}}$ in Eq. (5) can be considered as unity.

Then, it was demonstrated in Ref. [10] that the Gibson and Lanni ray-tracing method to compute the aberration function [11] is fully equivalent to the Török and Varga approach [6], so that the illumination PSF can be computed as:

$$
\begin{aligned}
I_{0 i l l}= & \int_{0}^{\alpha}\left(\cos \theta_{1}\right)^{1 / 2} \sin \theta_{1} J_{0}\left(k_{1}\left(x^{2}+y^{2}\right)^{1 / 2} \sin \theta_{1}\right) \\
& \times\left(t_{12 s} t_{23 s}+t_{12 p} t_{23 p} \cos \theta_{3}\right) \exp \left(i k_{0} O P D\right) d \theta_{1} \\
I_{1 i l l}= & \int_{0}^{\alpha}\left(\cos \theta_{1}\right)^{1 / 2} \sin \theta_{1} J_{1}\left(k_{1}\left(x^{2}+y^{2}\right)^{1 / 2} \sin \theta_{1}\right) \\
& \times t_{12 p} t_{23 p} \sin \theta_{3} \exp \left(i k_{0} O P D\right) d \theta_{1} \\
I_{2 i l l}= & \int_{0}^{\alpha}\left(\cos \theta_{1}\right)^{1 / 2} \sin \theta_{1} J_{2}\left(k_{1}\left(x^{2}+y^{2}\right)^{1 / 2} \sin \theta_{1}\right) \\
& \times\left(t_{12 s} t_{23 s}-t_{12 p} t_{23 p} \cos \theta_{3}\right) \exp \left(i k_{0} O P D\right) d \theta_{1}
\end{aligned}
$$

with: 


$$
\begin{aligned}
O P D & =n_{i} z \sqrt{1-\left(\frac{N A \rho}{n_{i}}\right)^{2}}-\left(\sqrt{1-\left(\frac{N A \rho}{n_{g}}\right)^{2}}-\left(\frac{n_{i}}{n_{g}}\right)^{2} \sqrt{1-\left(\frac{N A \rho}{n_{i}}\right)^{2}}\right) \\
& +n_{g} t_{g}\left(\sqrt{n_{g}}\right)^{2} \\
& -n_{g}^{*} t_{g}^{*}\left(\sqrt{\left.1-\left(\frac{n_{i}}{n_{g}^{*}}\right)^{*} \sqrt{1-\left(\frac{N A \rho}{n_{i}}\right)^{2}}\right)}\right. \\
& -n_{i}^{*} t_{i}^{*}\left(\sqrt{1-\left(\frac{N A \rho}{n_{i}^{*}}\right)^{2}}-\left(\frac{n_{i}}{n_{i}^{*}}\right)^{2} \sqrt{1-\left(\frac{N A \rho}{n_{i}}\right)^{2}}\right) \\
& -n_{s} t_{s}\left(\sqrt{1-\left(\frac{N A \rho}{n_{s}}\right)^{2}}-\left(\frac{n_{i}}{n_{s}}\right)^{2} \sqrt{1-\left(\frac{N A \rho}{n_{i}}\right)^{2}}\right)
\end{aligned}
$$

One has to compute the Optical Path Difference (OPD) with:

$\rho=n_{i} \sin \theta_{1} / N A$

NA : numerical aperture of the microscope objective

$\mathrm{z}$ : defocusing

$t_{s}:$ depth of the specimen under the cover glass

$n_{s}$ : index of refraction of the specimen

$t_{g}:$ thickness of the cover glass

$n_{g}:$ index of refraction of the cover glass

$t_{i}:$ thickness of the immersion medium layer

$n_{i}$ : index of refraction of the immersion medium

$n$ : index of refraction of the objective front lens

The parameters with an asterisk * are values for the design conditions of use of the objective, those without an asterisk are the actual ones [10,11]. The OPD is the sum of a defocus term, an aberration term describing the effect of using a non-design (thickness and refraction index) cover glass, a term describing the effect of the actual immersion medium and a term describing the spherical aberration induced when the index of refraction of the specimen differs from that of the immersion medium.

Now, analysis of Eq. (10) and of the expressions of $T_{s}^{\prime}$ and $T_{p}^{\prime}$ Eq. (12) shows that the total aberration function when observing the dipole has exactly the same form than the aberration 
function when focusing a wave for illuminating the dipole. As a consequence, the initial aberration function for detection may similarly be approximated as:

$$
\Psi_{\mathrm{det}}=\sum_{j=1}^{3} h_{j}\left(n_{j} \cos \theta_{j}^{\prime}-n_{j+1} \cos \theta_{j+1}^{\prime}\right)
$$

and the diffraction integrals for detection may also be computed using the Gibson and Lanni aberration function:

$$
\begin{aligned}
I_{0 \mathrm{det}}= & \int_{0}^{\alpha_{d}}\left(\cos \theta_{1}^{\prime}\right)^{-1 / 2} \sin 2 \theta_{d} J_{0}\left(k_{d}\left(x^{\prime 2}+y^{\prime 2}\right)^{1 / 2} \sin \theta_{d}\right) \\
& \times\left(t_{32 s} t_{21 s}+t_{32 p} t_{21 p} \cos \theta_{3}^{\prime}\right) \exp \left(-i k_{0} O P D^{\prime}\right) d \theta_{d} \\
I_{1 \operatorname{det}}= & \int_{0}^{\alpha_{d}}\left(\cos \theta_{1}^{\prime}\right)^{-1 / 2} \sin 2 \theta_{d} J_{1}\left(k_{d}\left(x^{\prime 2}+y^{\prime 2}\right)^{1 / 2} \sin \theta_{d}\right) \\
& \times t_{32 p} t_{21 p} \sin \theta_{3}^{\prime} \exp \left(-i k_{0} O P D^{\prime}\right) d \theta_{d} \\
I_{2 \operatorname{det}}= & \int_{0}^{\alpha_{d}}\left(\cos \theta_{1}^{\prime}\right)^{-1 / 2} \sin 2 \theta_{d} J_{2}\left(k_{d}\left(x^{\prime 2}+y^{\prime 2}\right)^{1 / 2} \sin \theta_{d}\right) \\
& \times\left(t_{12 s} t_{23 s}-t_{12 p} t_{23 p} \cos \theta_{3}^{\prime}\right) \exp \left(-i k_{0} O P D^{\prime}\right) d \theta_{d}
\end{aligned}
$$

With an OPD' simply computed with $\rho=n_{i} \sin \theta_{1}^{\prime} / N A$. We now have a rigorous and easy-touse model to compute the illumination $\mathrm{PSF}_{\text {ill }}$ for a confocal microscope, from which we can determine the induced dipole, depending on the fluorescence process (linear, quadratic or cubic being used in biology), as well as a rigorous and easy-to-use model to compute the detection $\mathrm{PSF}_{\text {det }}$ (which is the same for either a confocal or a classical microscope). Combining both permits to calculate the total confocal point spread function PSF $_{\text {conf }}$.

We have checked the validity of this approach in the three configurations commonly used for biological investigations (air-, oil- and water immersion objectives). In all cases, for the illumination as well as for the detection PSF, no differences were noticed when computing the curves with or without the hypothesis, which leads Eqs. $(14,15)$ and Eqs. $(17,18)$. This is due to the relatively small differences between the various indices of refraction (even in the case of an air to glass interface). 
As an illustration, Figure 2 shows the axial detection PSF (the axis Oz and O'z' of Fig. 2 being colinear) computed with Eqs. (18), assuming $\boldsymbol{p}_{\mathrm{e}}=\mathbf{c o n s t}=p_{\mathrm{ex}} \mathbf{i}_{\mathrm{x}}$, for a dipole immersed in a watery medium $\left(n_{\mathrm{s}}=1.33\right)$, at a depth of $50 \mu \mathrm{m}$, for an air immersion $\left(n_{\mathrm{i}}=n_{\mathrm{i}^{*}}=1\right)$ objective of numerical aperture NA=0.9 imaging at $\lambda=488 \mathrm{~nm}$ through a cover glass $\left(n_{\mathrm{g}}=n_{\mathrm{g}^{*}}=1.54\right.$, $t_{\mathrm{g}^{*}}=170 \mu \mathrm{m}$ ) of thickness $t_{\mathrm{g}}=120 \mu \mathrm{m}$ (curve (a)), $170 \mu \mathrm{m}$ (curve (b)) and $220 \mu \mathrm{m}$ (curve (c)). We show results for an air immersion objective because that case corresponds to the largest refractive index gap encountered in biological applications (glass/air interface). One maybe could not neglect the Fresnel reflection coefficients in Eq. (12) in that case, and as a consequence, Eqs. $(17,18)$ may fail. Figure 3 shows curve (c) of Figure 2, for a $220 \mu \mathrm{m}$ cover glass for which the highest aberrations are observed, computed with Eqs. (8) (upper curve (a)) and with Eqs. (18), neglecting the Fresnel reflection coefficients (lower curve (b)) (Data are displayed on a logarithmic scale, to highlight the lower intensity diffraction rings). The two curves are almost identical, with very tiny differences visible only in the higher order diffraction rings, of very low intensity and of no practical use. (For the sake of clarity, curve (a) has been multiplied by 10). This proofs the validity of our approach. The difference on the z-axis between Fig. 2 and Fig. 3 is because when using Eq. (18), one computes the PSF in an absolute reference frame centered at the geometrical position of the focal point, as in Ref. [10], while curves presented in Fig. 3 are displayed with the distance of the last interface from the unaberrated Gaussian focus as horizontal axis, as in Refs. [6,8].

Curves in the same conditions as for Fig. 2 but for the illumination PSF have been published in Ref. [10]. In that work, the validity of Eqs. (15) (compared to Eqs. (3)) was demonstrated. To conclude, Equations $(15,18)$ always constitute excellent approximations to compute the illumination and detection PSFs in a confocal microscope, respectively. Explicitly introducing the objective's design and actual conditions of use, they therefore constitute a very helpful method for modeling the image formation process in biological microscopy. 


\section{Crystallography and semiconductor applications}

The situation could be different in crystallography. This is in particular the case for observation into diamond ( $\mathrm{n}=2.418$ at $\lambda=633 \mathrm{~nm}$ in the visible) or into silicon $(\mathrm{n}=3.44$ at $\lambda=1.3 \mu \mathrm{m}$ in the infrared where it is transparent), because of the very high index of refraction. Special objectives with very high NA, using special immersion oil have been developed for crystallography. However, wafer inspection imposes to work in a clean environment, and air immersion objectives are mandatory in that case.

Because of the high index of refraction, Eqs. $(15,18)$ may fail. We would like to attract the attention of the reader to the fact that for a single interface, the Fresnel coefficients for a stratified medium $T_{2 s, p}$ and $T_{2 s, p}^{\prime}$ (Eqs. $\left.(5,12)\right)$ of course reduce to usual Fresnel coefficients given by Eqs. $(6,13)$. As a consequence, our approach including the Gibson and Lanni aberration function into vectorial models for illumination or detection is always valid for single interfaces, as for example for observation into diamond as presented in Ref. [8]. The sole thing to do is simply to put the value of the cover glass thickness to zero $\left(t_{g}=t_{g}^{*}=0\right)$ so that its associate aberration term in Eq. (16) is null (this remark also holds for no cover glass objectives in biology).

We consider now the peculiar case of a diamond layer deposited onto a silicon substrate. This configuration may be interesting for semiconductor applications because of the intrinsic properties of diamond, which can be either an isolator, a semiconductor or a conductor, depending on its doping. Furthermore, diamond is an excellent heat conductor, and is transparent in the infrared, which make it interesting for detector applications.

At $\lambda=1.3 \mu \mathrm{m}$ in the infrared, we therefore consider an air/diamond/silicon sandwich, for which two very large index of refraction gaps are present $\left(n_{1}=1, n_{2}=2.420, n_{3}=3.44\right)$. The denominator of the expression for the transmission coefficient $\left(1+r_{12 s, p} r_{23 s, p} \exp (-2 i \beta)\right)$ can indeed be very different from one in that case. 
Figure 4(a) shows the results obtained when computing the axial PSF for illumination with Eqs. (3) and Eqs. (15) (solid lines) and for detection, with Eqs. (8) and Eqs. (18) (dashed lines), considering a N.A. $=0.8$ objective, at $\lambda=1.3 \mu \mathrm{m}$, and for an observation at $50 \mu \mathrm{m}$ below the diamond/silicon interface, with a $1 \mu \mathrm{m}$ thick diamond layer.

A commonly used approximation for confocal microscope is to consider that the illumination and detection PSF are identical. This is not always true, as shown in Ref. [8] and by Fig. 4(a), the illumination and the detection PSF being slightly different. Furthermore differences do appear when computing the curves with Eqs. $(3,8)$ (thick lines), or with the approximate Eqs. $(15,18)$ (thin lines).

Figure 4(b) shows results for a $20 \mu \mathrm{m}$ thick diamond layer (all other parameters being identical): discrepancies are clearly visible in that case between the curves computed with the exact and with the approximate diffraction integrals.

By coincidence, the curves computed for the confocal case, shown in Figure 4(c), are almost identical (differences are pointed out by arrows), because the errors in the illumination PSF and the detection PSF mostly compensate (throughout this work, we use the usual simplification considering that the confocal PSF is obtained by multiplying the excitation PSF by the detection PSF, $\left.\mathrm{PSF}_{\mathrm{conf}}=\mathrm{PSF}_{\mathrm{ill}} * \mathrm{PSF}_{\mathrm{det}}\right)$. As a consequence, our model, while not being strictly valid for computing the illumination PSF and the detection PSF, may still gives very good results for the confocal PSF, but should be used with care.

Note that for Figs. 4(a)-(c), in order to facilitate the comparison, all curves are displayed in the same reference frame, centered at the geometrical position of the focal point.

To conclude, for single interface configurations, our model is always valid, for example for crystallographic applications, or for wafer inspection. The model is still good, but less precise when considering thick layered specimen, displaying two large index of refraction gaps, as the given air/diamond/silicon example. 
As a final remark, we would like to mention the following fact: at a given depth, we observed that for the considered N.A. $=0.8$ objective and at the same given depth, the PSF in silicon in the infrared at $\lambda=1.3 \mu \mathrm{m}$ is less aberrated than the PSF in diamond in the visible at $\lambda=488 \mathrm{~nm}$, despite the much larger index of refraction. This fact can be explained easily with our model. The phase aberration is given by $k_{0} O P D$, and analysis of Eq. (16) shows that OPD is essentially proportional to $\mathrm{n}_{\mathrm{s}}$. While the index of refraction of silicon is $3.44,1.42$ times larger than that of diamond, the wave vector at $\lambda=1.3 \mu \mathrm{m}$ is 2.66 times smaller than for $\lambda=488 \mathrm{~nm}$, so that the overall aberration factor is finally lower. This has interesting consequences for biological applications.

\section{Multi-photon excitation}

Bi-photon excitation was predicted in 1931 [12], and first demonstrated in 1961 [13], but it was not until 1990 that it was successfully applied to microscopy [14]. It has now become a practical tool in many fields of biology [15]. Tri-photon excitation is also possible [16].

Bi-photonic excitation permits good imaging of thick samples for two main reasons. First, it offers optical sectioning without the need of a confocal setup, therefore allowing a more efficient collection of the (often weak) fluorescent signal. Secondly, using near infrared radiation to excite fluorophores permits a higher penetration depth, because of the muchreduced scattering of the incident photons. While these arguments are of course correct, we would like to mention another reason.

Figure 5(a) shows PSF ill computed for an oil-immersion objective with N.A.=1.4, at $\lambda=450 \mathrm{~nm}$ (1-photon excitation), and at $\lambda=900 \mu \mathrm{m}$ (2-photon process), and for a depth of $0 \mu \mathrm{m}$ and $100 \mu \mathrm{m}$ into a watery medium $\left(\mathrm{n}_{\mathrm{s}}=1.33\right)$, through a $170 \mu \mathrm{m}$ cover glass. We suppose a non- or weakly scattering medium. The n-photon fluorescence PSF at wavelength $\lambda$ is proportional to the $n^{\text {th }}$-power of the 1 -photon PSF. 
Just below the cover glass, the resolution of a (non-confocal, or confocal) 2-photon microscope is actually slightly worse than that of a (non-confocal, or confocal) 1-photon microscope. This is because squaring the PSF does not compensate for its widening by a factor two because of the doubled wavelength.

However, at a depth of $100 \mu \mathrm{m}$, the 1-photon excitation PSF is much more degraded than its 2-photon homolog. So, because longer wavelength beams are less affected by spherical aberrations, the resulting illumination PSF $_{\text {ill }}$ is less blurred with a 2-photon excitation. First, this has for effect to even further reduce the photobleaching compared to 1-photon excitation. Secondly, the lower spherical aberration, together with the reduced scattering, also contributes to concentrate more photons in a small volume, therefore increasing the 2-photon excitation efficiency.

We consider now fluorescence detection at $600 \mathrm{~nm}$ and the effects of confocalization. Figure 5(b) shows the detection $\mathrm{PSF}_{\text {det }}$, at $600 \mathrm{~nm}$ (gray line) and the total $\mathrm{PSF}_{\text {conf }}$ $\left(\mathrm{PSF}_{\mathrm{conf}}=\mathrm{PSF}_{\mathrm{ill}} * \mathrm{PSF}_{\mathrm{det}}\right)$ of a 1 -photon confocal microscope (thick solid line) and of a confocal 2-photon microscope (thick dashed line), as well as the non-confocal 2-photon microscope PSF (thin dashed line)

The 1-photon confocal microscope has a longitudinal resolution (defined at FWHM) of $2.3 \mu \mathrm{m}$, while for the confocal 2-photon PSF a FWHM of $2.2 \mu \mathrm{m}$ is obtained. Contrary to an observation just below the surface, the 2-photon microscope now has a better resolution than the 1-photon microscope. Note that the non-confocal 2-photon microscope has a longitudinal resolution of $3.2 \mu \mathrm{m}$ only. This gives a strong argument in favor of the systematic confocalization of 2-photon microscopes, despite the cost to pay of an actual loss of photon detection efficiency. Note also that the focal shift is different for the 2-photon and the 1photon microscopes [17]. The difference is however in practice very weak $(1 \mu \mathrm{m}$ at a depth of $100 \mu \mathrm{m})$. 


\section{Conclusion}

We have given simple models to compute both the illumination and the detection Point Spread Functions of optical microscopes focusing through a stratified medium. By combining rigorous vectorial approaches with a simple ray-tracing method to compute the aberration function, we explicitly introduces experimental and design acquisition parameters, as recommended by the objective manufacturer. This should contribute to the adoption of rigorous vectorial models for computing microscope PSFs for modeling the image formation process, or in view of deconvolution in order to improve the acquired data.

For biological observation, the high accuracy of this approach has been established for conventional and confocal microscope. The model is also valid for multi-photon excitation. When large differences in the refractive index are present, as in crystallography, our method still gives good results, but should be used with more care.

Our model may also permit to better model the illumination and detection PSFs of less common configurations, like 4-Pi microscopy or STED microscopy, which have proven to deliver an unsurpassed resolution [18-20]. They are for this reason even more sensitive to aberrations, but also because these methods rely on the precise interference of two counterpropagating illumination beam (4-Pi), or on the precise superposition of an excitation beam and a STED beam (STED microscopy), which have different wavelengths. 


\section{References}

[1] S.W. Hell, G. Reiner, C. Cremer, and E.H.K. Stelzer, J. Microsc. (Oxford) 169, 391$405(1993)$

[2] P. Török, P. Varga, Z. Laczik, and G.R. Booker, J. Opt. Soc. Am. A 12, 325-332 (1995)

[3] P. Török, P. Varga, and G.R. Booker, J. Opt. Soc. Am. A 12, 2136-2144 (1995)

[4] P. Török, P. Varga, A. Konkol, and G.R. Booker, J. Opt. Soc. Am. A 13, 2232-2238 (1996)

[5] A. Egner and S.W. Hell, J. Microsc. (Oxford) 193, 244-249 (1999)

[6] P. Török and P. Varga, Appl. Opt. 36, 2305-2312 (1997)

[7] P. Török, Opt. Lett. 25, 1463-1465 (2000)

[8] O. Haeberlé, M. Ammar, H. Furukawa, K. Tenjimbayashi and P. Török, Opt. Exp. 11, 2964-2969 (2003) http://www.opticsexpress.org/abstract.cfm?URI=OPEX-11-22-2964

[9] M. Minsky, Scanning 10, 128-138 (1988)

[10] O. Haeberlé, Opt. Comm. 216, 55-63 (2003)

[11] S.F. Gibson and F. Lanni, J. Opt. Soc. Am. A 8, 1601-1613 (1991)

[12] M. Göpper-Meyer, Ann. Phys. 9, 273-294 (1931)

[13] W. Kaiser and C. Garret, Phys, Rev. Lett. 7, 229-231 (1961)

[14] W. Denk, J.H. Strickler and W.W. Webb, Science 248, 73-75 (1990)

[15] K. König, J. Microsc. 200, 83-104 (2000)

[16] M. Schrader, K. Bahlmann and S.W. Hell, Optik 104, 116-124 (1997)

[17] A. Egner, M. Schrader and S.W. Hell, Opt. Comm. 153, 211-217 (1998)

[18] S.W. Hell and E.H.K Stelzer., Opt. Comm. 93, 277-282 (1992)

[19] M. Dyba and S.W. Hell, Phys. Rev. Lett. 88, 163901 (2002)

[20] V. Westphal, L. Kastrup and S.W. Hell, Appl. Phys. B 77, 377-380 (2003) 


\section{Figure Captions}

Figure 1: Sketch of a confocal microscope. The excitation beam is focused by the objective at a point $\mathrm{O}$, origin of the $(\mathrm{x}, \mathrm{y}, \mathrm{z})$ reference frame. A dipole in the nearby, located at point $\mathrm{O}^{\prime}$, origin of the $\left(\mathrm{x}^{\prime}, \mathrm{y}^{\prime}, \mathrm{z}^{\prime}\right)$ reference frame, is excited and emits fluorescence light, which is collected by the objective and focused onto the detection pinhole. With a large detector and uniform illumination, one obtains a wide-field microscope. For clarity, the distance between $\mathrm{O}$ and $\mathrm{O}$ ' has been largely amplified and only one ray for illumination and one ray for detection are depicted.

Figure 2: Optical axis profile of the detection point spread function for an air immersion $\left(n_{\mathrm{i}}=n_{\mathrm{i}^{*}}=1\right)$ objective of numerical aperture NA$=0.9$ imaging at $\lambda=488 \mathrm{~nm}$ a specimen at a depth of $50 \mu \mathrm{m}$ in a watery medium $\left(n_{\mathrm{s}}=1.33\right)$ through a cover glass $\left(n_{\mathrm{g}}=n_{\mathrm{g}^{*}}=1.54, t_{\mathrm{g}^{*}}=170 \mu \mathrm{m}\right)$ of thickness $120 \mu \mathrm{m}$ (curve (a)), $170 \mu \mathrm{m}$ (curve (b)) and $220 \mu \mathrm{m}$ (curve (c)). These curves are homologues to those published in Ref. [10] for the illumination point spread function.

Figure 3: Curve (c) of Fig. 2, computed with the exact diffraction integrals Eqs. (8) (solid line, $\mathrm{x} 10$ for the sake of clarity) and their approximate form Eqs. (18) (dashed line). The curves are almost identical, proving the validity of our approach for biological applications. 
Figure 4: Optical axis profile of the detection $\mathrm{PSF}_{\mathrm{det}}$ and illumination $\mathrm{PSF}_{\mathrm{ill}}$, for an air immersion objective of numerical aperture $\mathrm{NA}=0.8$, imaging at $\lambda=1.3 \mu \mathrm{m}, 50 \mu \mathrm{m}$ into silicon through a diamond layer. (a): $1 \mu \mathrm{m}$ thickness diamond layer (b): $20 \mu \mathrm{m}$ thickness diamond layer. (c): confocal PSF. Note the difference between PSF $_{\text {det }}$ and $\mathrm{PSF}_{\mathrm{ill}}$ and between the curves computed with the exact diffraction integrals Eqs. (8) and their approximate form Eqs. (18). The approximate confocal PSF is however still a good approximation of the exact one.

Figure 5: (a) Comparison of the illumination PSF $_{\text {ill }}$ for a one-photon excitation at $450 \mathrm{~nm}$ and a 2-photon excitation at $900 \mathrm{~nm}$ for an oil immersion objective with N.A. $=1.4$ imaging into a watery medium through a $170 \mu \mathrm{m}$ cover glass at $0 \mu \mathrm{m}$ and $100 \mu \mathrm{m}$ below the surface. (b) Comparison of the confocal PSF conf for one-photon (thick solid line) and two-photon excitation (dashed solid line), with a fluorescence detection $\mathrm{PSF}_{\operatorname{det}}$ at $\lambda=600 \mathrm{~nm}$ (gray line). Note the slight shift in the position of the maxima of the confocal point spread functions for 1-photon and 2-photon excitations. 


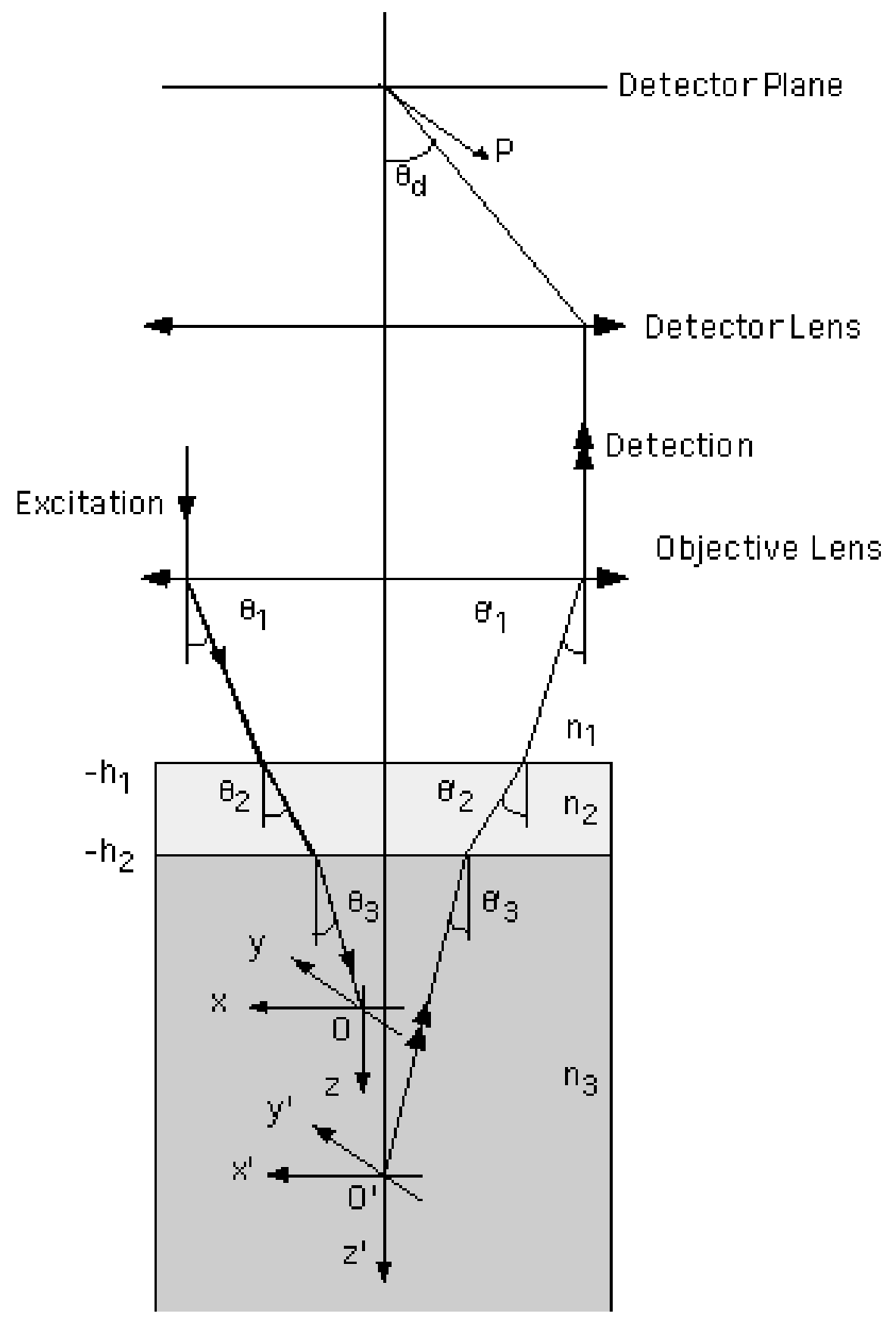

O. Haeberlé "Focusing of light..." Fig. 1 


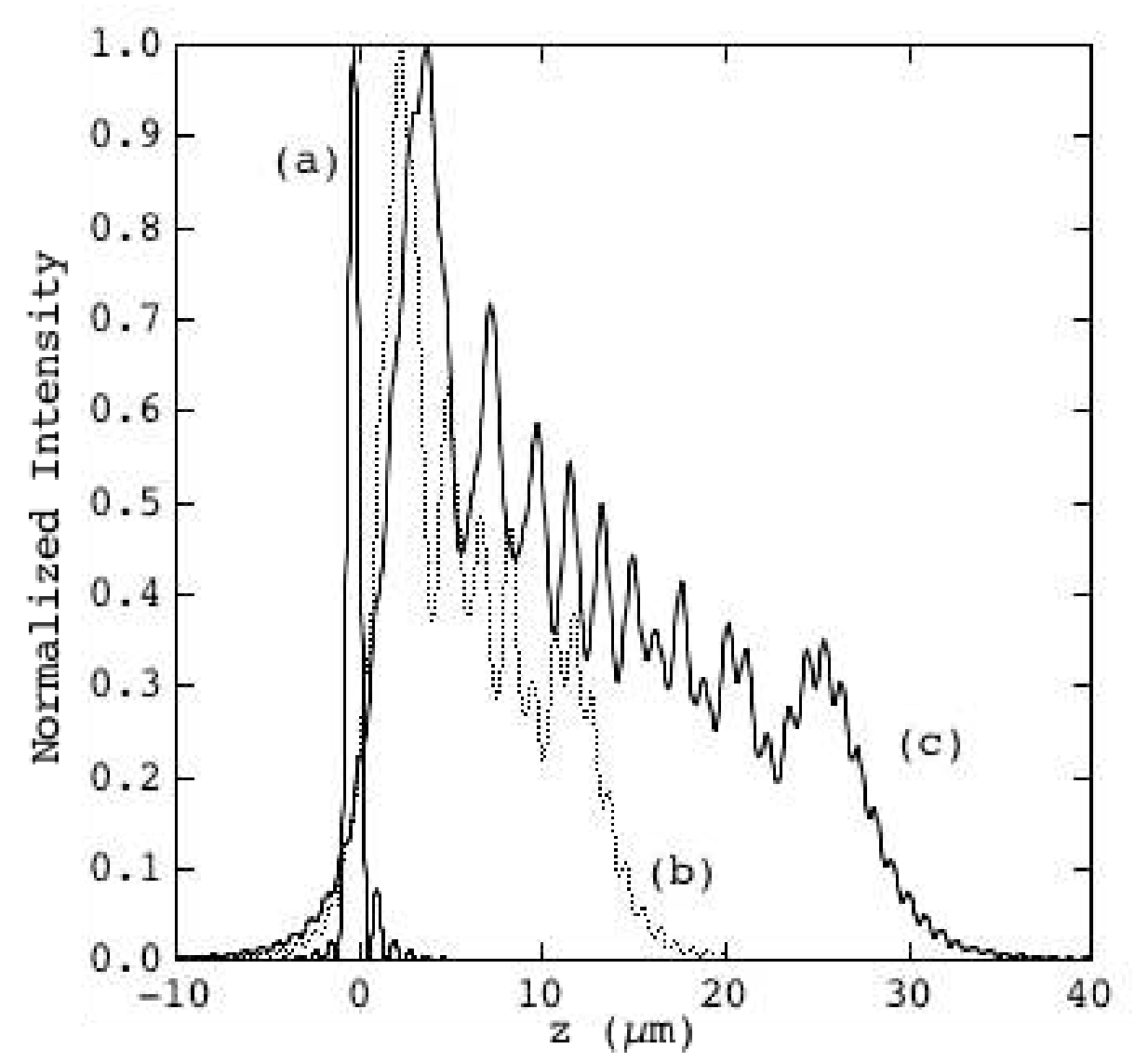

O. Haeberlé "Focusing of light..." Fig. 2 


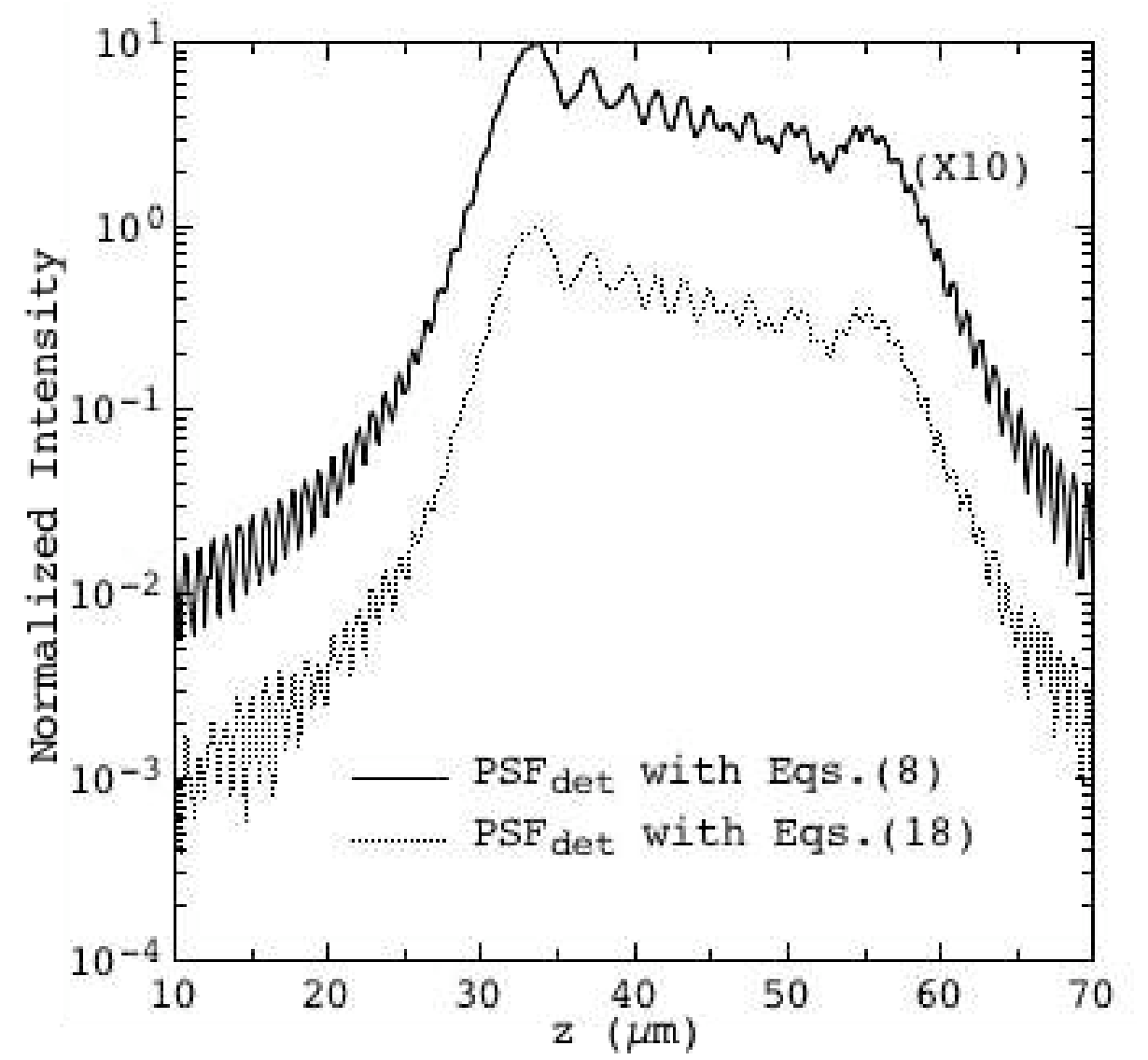

O. Haeberlé "Focusing of light..." Fig. 3 


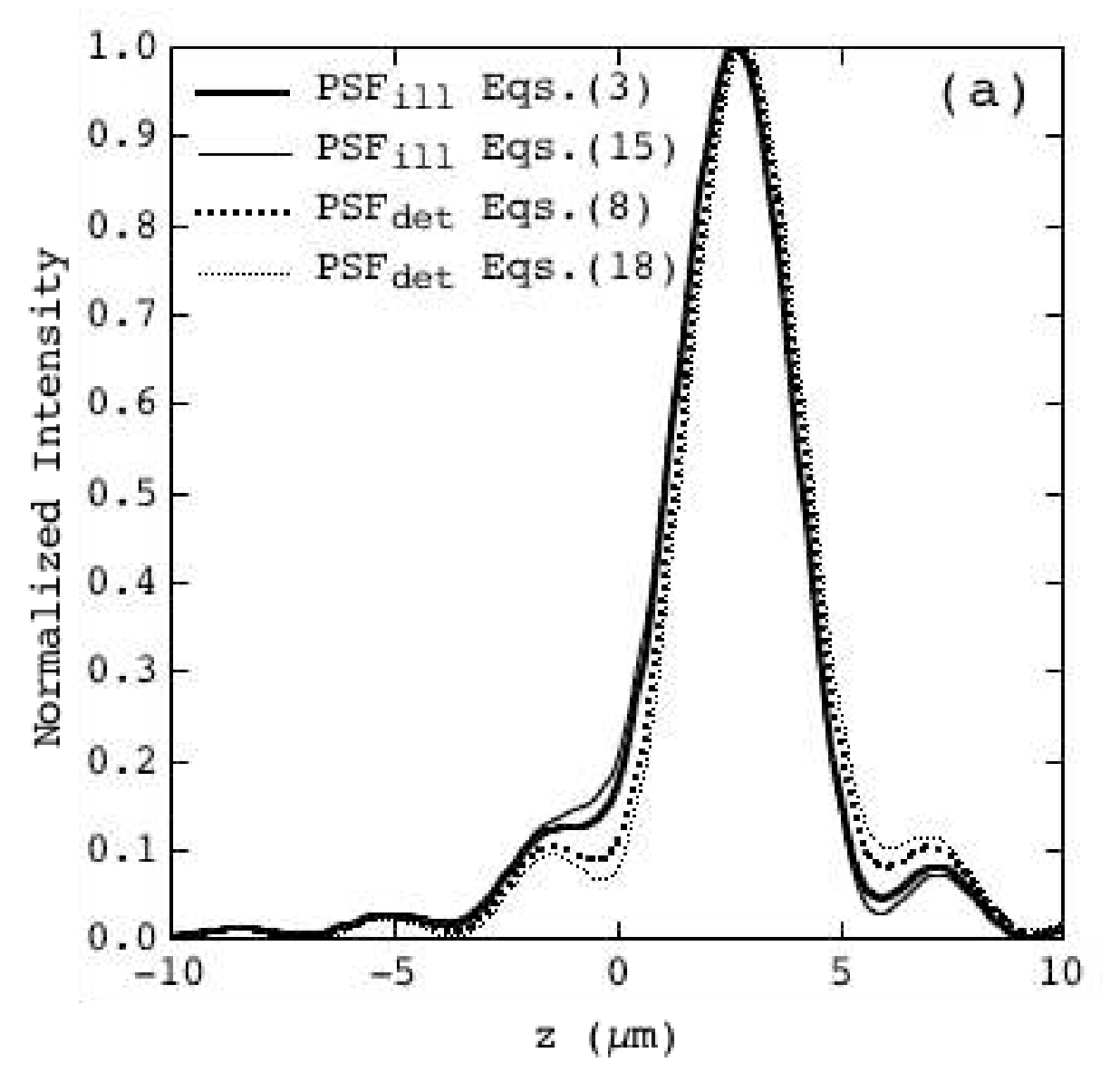

O. Haeberlé "Focusing of light..." Fig. 4(a) 


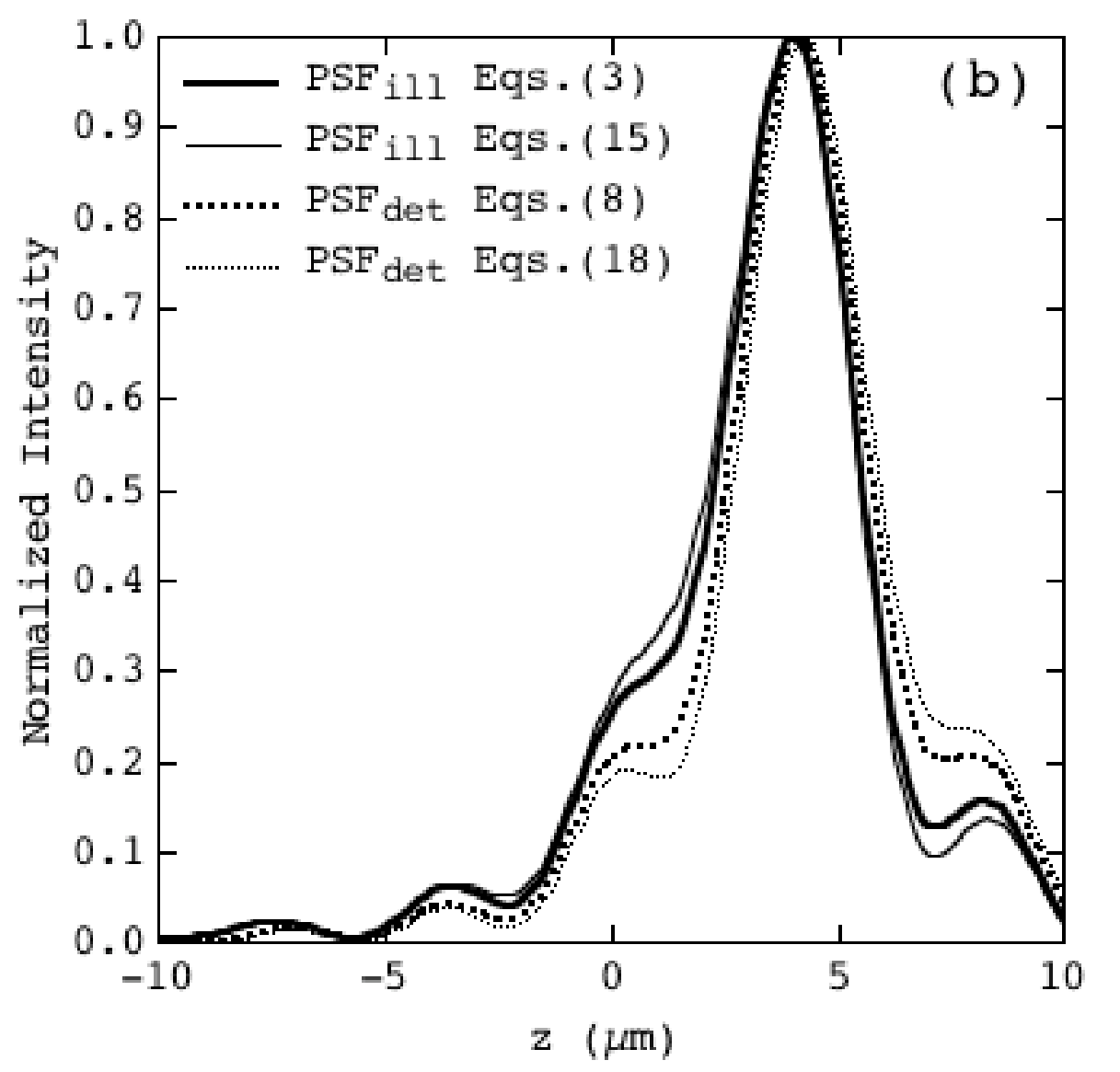

O. Haeberlé "Focusing of light..." Fig. 4(b) 


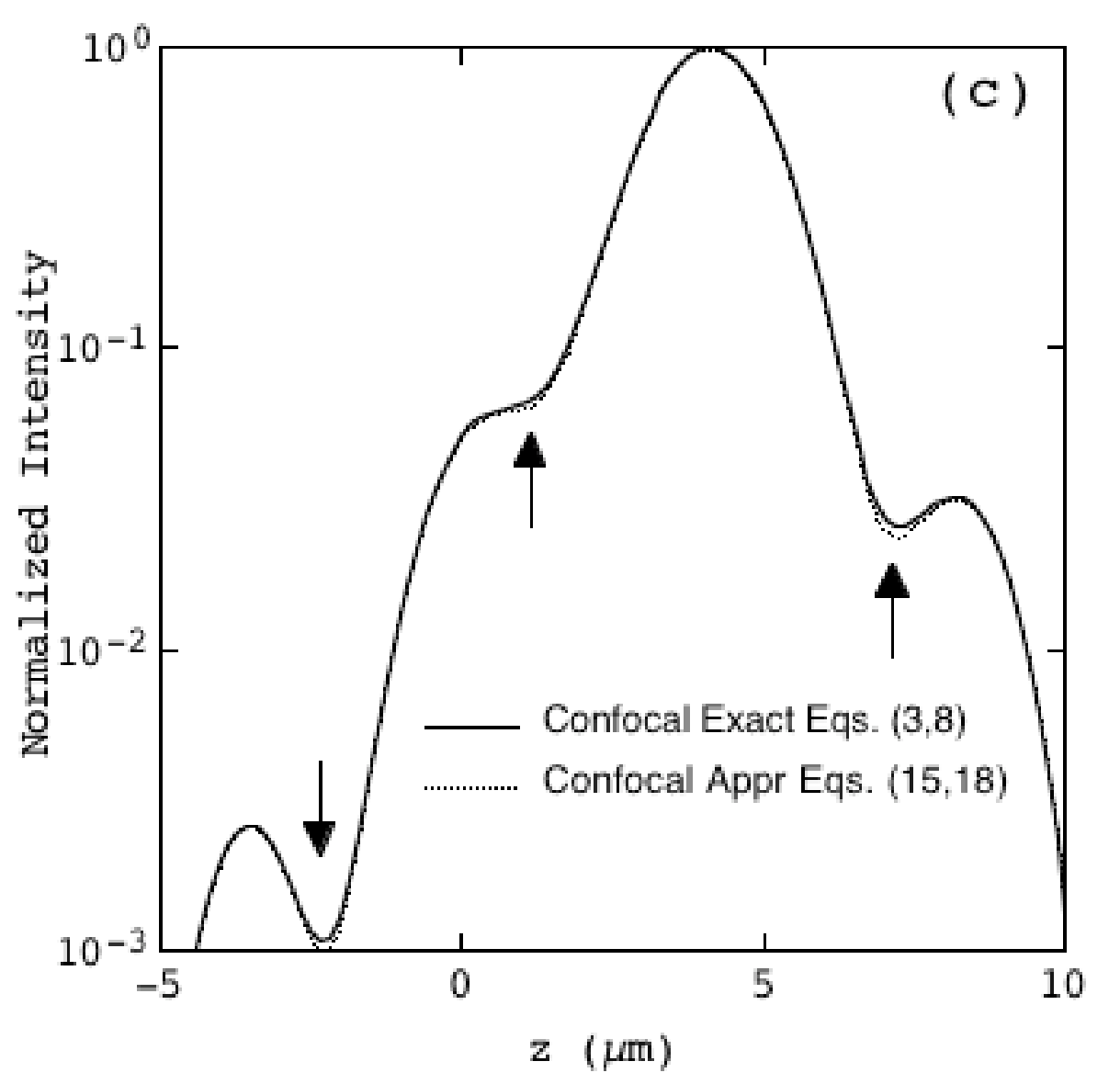

O. Haeberlé "Focusing of light..." Fig. 4(c) 


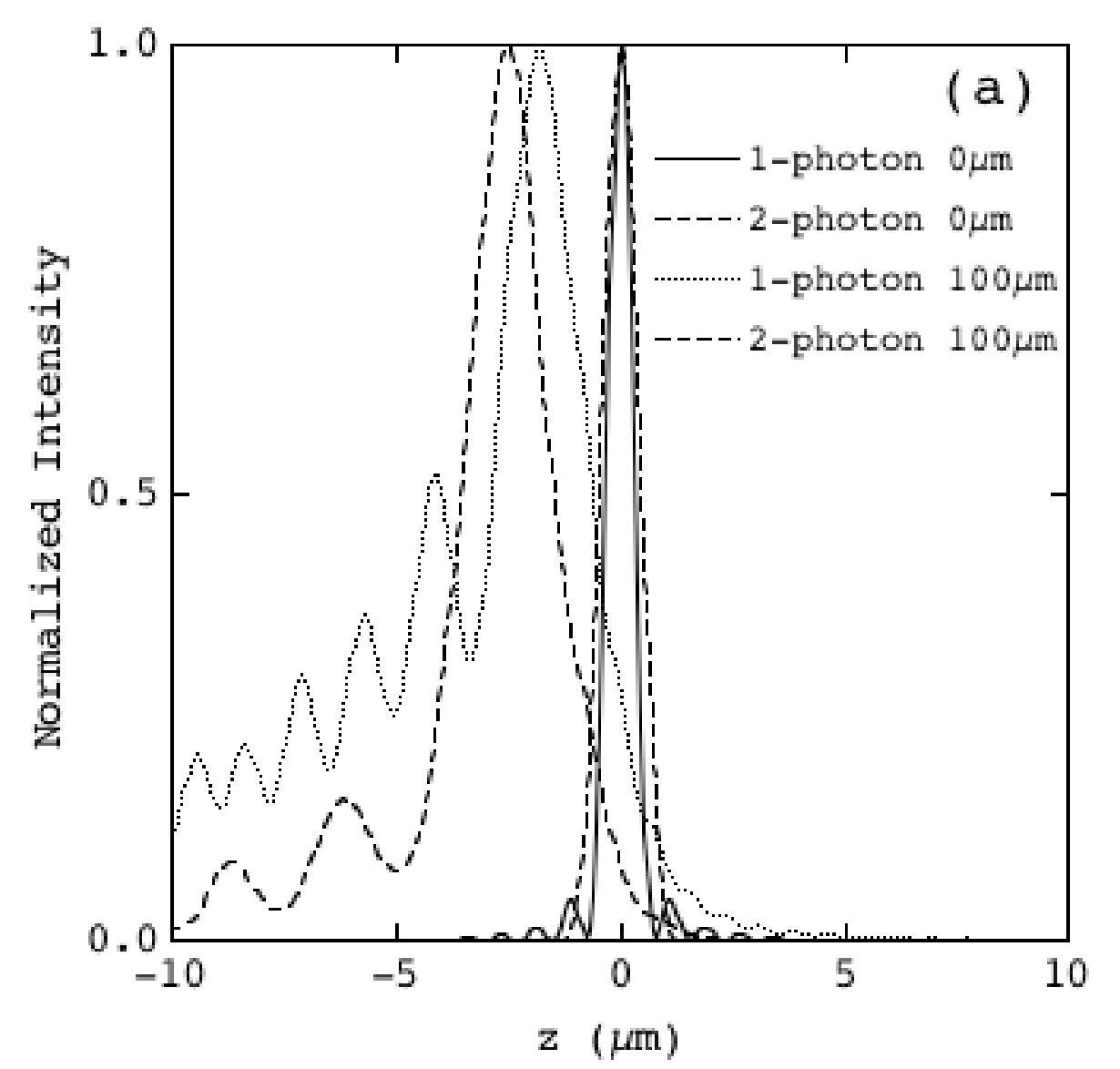

O. Haeberlé "Focusing of light...” Fig. 5(a) 


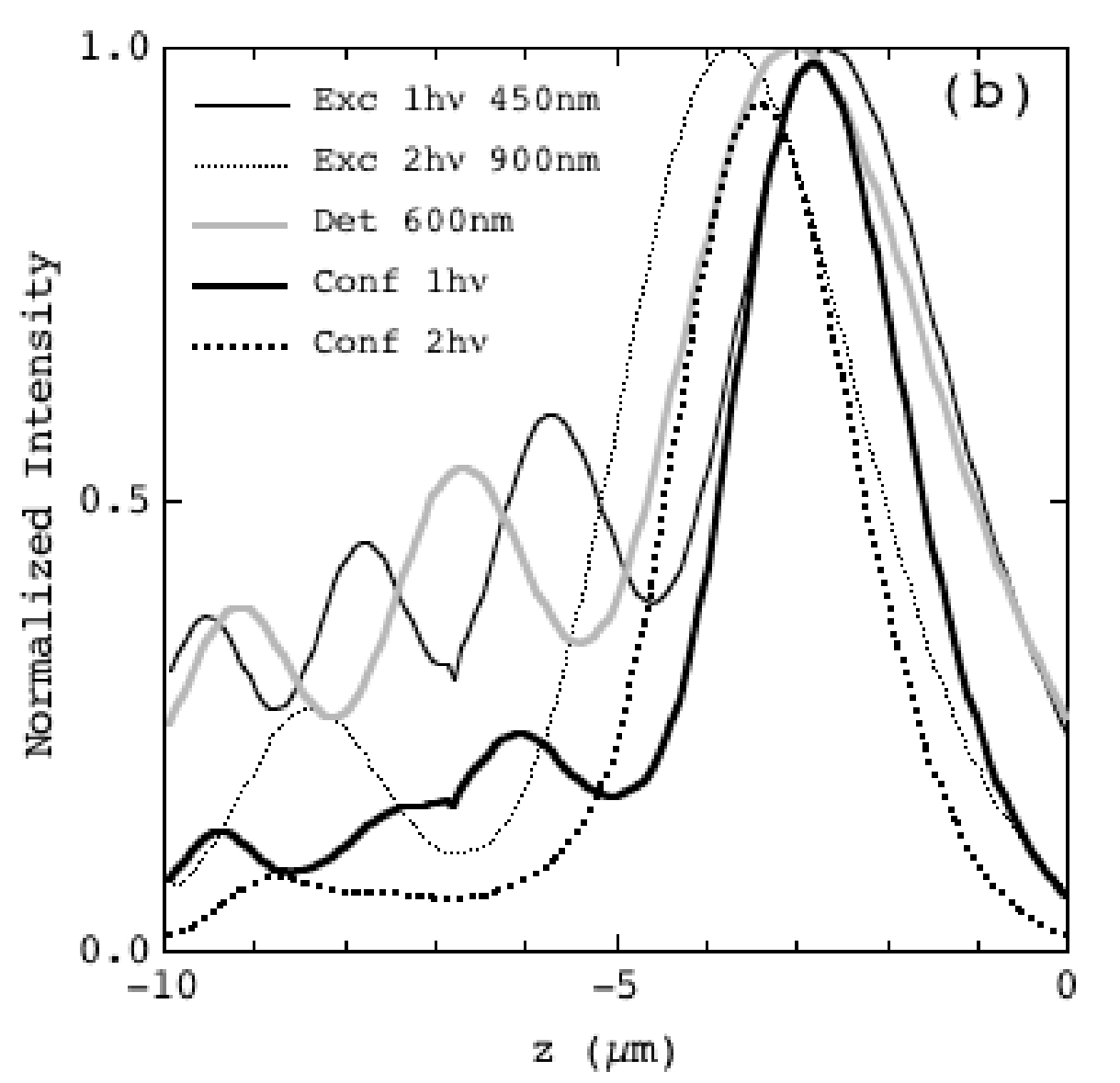

O. Haeberlé "Focusing of light..." Fig. 5(b) 